\title{
Heparanase and Thrombin: Common Signalling Pathways in Melanoma Cells?
}

\author{
Georg Breier ${ }^{1}$ \\ ${ }^{1}$ Division of Medical Biology, Department of Psychiatry and \\ Psychotherapy, Faculty of Medicine Carl Gustav Carus, TU Dresden, \\ Dresden, Germany \\ Thromb Haemost 2018;118:1688-1689.
}

Address for correspondence Georg Breier, PhD, Division of Medical Biology, Department of Psychiatry and Psychotherapy, Faculty of Medicine Carl Gustav Carus, TU Dresden, 01307 Dresden, Germany (e-mail: georg.breier@uniklinikum-dresden.de).
Heparanase (HPSE) is a carbohydrate-processing enzyme that degrades heparan sulphate side chains of extracellular matrix and cell surface proteoglycans. ${ }^{1}$ HPSE can be expressed by cancer cells of different origin and has been implicated in various aspects of tumour progression and metastasis. $^{1,2}$ Through remodelling of the extracellular matrix, HSPE facilitates motility, invasion and the metastatic spread of malignant cells. Another consequence of heparan sulphate degradation is the release of extracellular matrixbound cytokines, including the heparin-binding isoforms of vascular endothelial growth factor (VEGF) which were secreted by tumour and stromal cells. The indirect proangiogenic activity of HPSE is further enhanced by its capability to stimulate the cellular production of VEGF. Clinically, HPSE expression correlates with poor prognosis, and HPSE inhibitors may therefore have potential as anticancer agents. ${ }^{1,3}$

While the influence of HPSE on tumour progression and angiogenesis is well documented, the mechanisms through which HSPE exerts its direct action on cells are only partially understood. Certain effects of HPSE, notably the abovementioned up-regulation of VEGF by tumour cells, do not require its enzymatic activity and may therefore be mediated by a cellular signalling receptor. ${ }^{4}$ Yet, the nature of this putative HPSE receptor remained elusive.

In this issue of Thrombosis and Haemostasis, Hoß et al report remarkable parallels between HSPE- and thrombininduced responses in human melanoma cells. ${ }^{5}$ In malignancies, thrombin is generated as a result of local tissue factor exposure on tumour cells and subsequent activation of the coagulation cascade. Thrombin can induce HPSE release from platelets and VEGF expression in tumour cells via a unique mechanism involving the protease-activated receptor-1 (PAR-1), a member of the G-protein coupled receptor (GPCR) superfamily. ${ }^{6,7}$ In their study, Hoß et al first show that both, the latent (enzymatically inactive) form of HPSE and a thrombin receptor agonist, TRAP-6, stimulate MV3 melanoma cells to release VEGF and another pro-angiogenic protein, cysteine-rich 61 . Cellular signalling was investigated by applying the elegant label-free dynamic mass redistribution (DMR) technology which allows for real-time detection of cellular responses in living cells. ${ }^{8}$ The authors demonstrate that latent HPSE evoked a characteristic DMR response. Substances that disrupt GPCR signalling, inhibit PAR-1 activity, or antagonize latent HPSE, interfered with the DMR signature, indicating the specificity of the response. The biological relevance of the DMR analysis was underlined by the observation that PAR-1 blockade interfered with thrombin- or HPSE-induced VEGF release.

Taken together, despite certain differences that became apparent in the study, novel evidence supports the hypothesis that latent HPSE and thrombin share a common signalling pathway involving the thrombin receptor, PAR-1. Although definitive proof of this hypothesis will require biochemical evidence for the physical interaction of HPSE with the putative receptor, the findings of the study add an interesting novel perspective on the complex interplay of HPSE, thrombin and other components of the haemostatic system in pro-angiogenic signalling in tumours.

Conflict of Interest

None.

\section{References}

1 Vlodavsky I, Singh P, Boyango I, et al. Heparanase: from basic research to therapeutic applications in cancer and inflammation. Drug Resist Updat 2016;29:54-75

2 Götte M, Yip GW. Heparanase, hyaluronan, and CD44 in cancers: a breast carcinoma perspective. Cancer Res 2006;66(21):10233-10237 received

September 4, 2018

accepted

September 4, 2018 (c) 2018 Georg Thieme Verlag KG Stuttgart · New York
DOI https://doi.org/

10.1055/s-0038-1673329. ISSN 0340-6245. 
3 Crispel Y, Axelman E, Tatour M, et al. Peptides inhibiting heparanase procoagulant activity significantly reduce tumour growth and vascularisation in a mouse model. Thromb Haemost 2016; 116(04):669-678

4 Zetser A, Bashenko Y, Edovitsky E, Levy-Adam F, Vlodavsky I, Ilan $\mathrm{N}$. Heparanase induces vascular endothelial growth factor expression: correlation with p38 phosphorylation levels and Src activation. Cancer Res 2006;66(03):1455-1463

5 HoßSG, Grundmann M, Benkel T, et al. Proangiogenic effects of latent heparanase and thrombin receptor mediated pathways - do they share a common ground in melanoma cells? Thromb Haemost 2018
6 Tsopanoglou NE, Maragoudakis ME. Thrombin's central role in angiogenesis and pathophysiological processes. Eur Cytokine Netw 2009;20(04):171-179

7 Tatour M, Shapira M, Axelman E, et al. Thrombin is a selective inducer of heparanase release from platelets and granulocytes via protease-activated receptor-1. Thromb Haemost 2017;117(07): 1391-1401

8 Schröder R, Schmidt J, Blättermann S, et al. Applying label-free dynamic mass redistribution technology to frame signaling of $G$ protein-coupled receptors noninvasively in living cells. Nat Protoc 2011;6(11):1748-1760 